\title{
Latent class evaluation of three serological tests for the diagnosis of human brucellosis in Bangladesh
}

\author{
A. K. M. A. Rahman ${ }^{1,2,3^{*}}$ (D, C. Saegerman ${ }^{2}$ and D. Berkvens ${ }^{3}$
}

\begin{abstract}
Background: A Bayesian latent class evaluation was used to estimate the true prevalence of brucellosis in livestock farmers and patients with prolonged pyrexia (PP) and to validate three conditionally dependent serological tests: indirect ELISA (iELISA), Rose Bengal Test (RBT), and standard tube agglutination (STAT). A total of 335 sera from livestock farmers and 300 sera from PP patients were investigated.

Results: The true prevalence of brucellosis in livestock farmers and PP patients was estimated to be $1.1 \%$ (95\% credibility interval (Crl) $0.1-2.8)$ and $1.7 \%(95 \% \mathrm{Crl} 0.2-4.1)$, respectively. Specificities of all tests investigated were higher than $97.8 \%$ (95 \% Crl 96.1-99.9). The sensitivities varied from $68.1 \%$ (95 \% Crl 54.5-80.7) to $80.6 \%$ (95 \% Crl 63.6-93.8). The negative predictive value of all the three tests in both populations was very high and more than 99. $5 \%$ (95 \% Crl 98.6-99.9). The positive predictive value (PPV) of all three tests varied from $27.9 \%$ (95\% Crl 3.6-62.0) to $36.3 \%$ (95 \% Crl 5.6-70.5) in livestock farmers and $39.8 \%$ (95\% Crl 6.0-75.2) to $42.7 \%$ (95\% Crl 6.4-83.2) in patients with PP. The highest PPV were $36.3 \%$ for iELISA and $42.7 \%$ for RBT in livestock farmers and pyrexic patients, respectively.
\end{abstract}

Conclusions: In such a low prevalence scenario, serology alone does not help in diagnosis and thereby therapeutic decision-making. Applying a second test with high specificity and/or testing patients having history of exposure with known risk factors and/or testing patients having some clinical signs and symptoms of brucellosis may increase the positive predictive value of the serologic tests.

Abbreviations: Crl, Credibility interval; DIC, Deviance Information Criterion; iELISA, Indirect ELISA; MMC, Mymensingh Medical College; NPV, Negative predictive value; PP, Prolonged pyrexia; PPV, Positive predictive value; RBT, Rose Bengal Test; sLPS, Anti-Brucella smooth lipopolysaccharide; STAT, Standard tube agglutination test

\section{Background}

Brucellosis is a bacterial zoonosis affecting both human and animal health [1]. It is an occupational hazard for livestock farmers, milkmen, butchers, hired animal caretakers, and veterinarians [2]. Fever, sweating, fatigue, headache, and joint pain are important non-specific symptoms of brucellosis in humans. Brucellosis in humans is often misdiagnosed due to its unspecific

\footnotetext{
* Correspondence: arahman_med@bau.edu.bd

${ }^{1}$ Department of Medicine, Bangladesh Agricultural University, Mymensingh 2200, Bangladesh

${ }^{2}$ Research Unit of Epidemiology and Risk Analysis applied to the Veterinary Sciences (UREAR-ULg), Department of Infectious and Parasitic Diseases,

Faculty of Veterinary Medicine, University of Liège, Liège, Belgium

Full list of author information is available at the end of the article
}

clinical symptoms similar to that of other endemic pyrexic diseases like tuberculosis, malaria, typhoid, or rheumatic fever. Several sero-prevalence studies from Bangladesh indicate that the apparent prevalence of brucellosis in risk groups varies from 4.4 to $12.8 \%$ [3-5]. The Rose Bengal Test (RBT), standard tube agglutination (STAT), and ELISA either alone or in combination were used for those studies. None of these tests is perfect, and thus, they cannot be used to estimate true prevalences. In the absence of a reasonable gold standard test, simultaneous estimation of true prevalence and test validation can be performed by applying multiple diagnostic tests to every individual using a Bayesian latent class analysis framework allowing the combination of test results and external information [6-8]. While 
evaluating the results of multiple diagnostic tests, it is essential to consider whether or not the tests can be assumed conditionally independent of each other given the true disease status. Assuming conditional independence may lead to biased estimates for test characteristics if the tests are conditionally dependent $[9,10]$. As indirect ELISA (iELISA), RBT, and STAT are based on the same biological phenomenon [11], i.e., detection of anti-Brucella smooth lipopolysaccharide (sLPS) antibodies, they can primarily be considered as conditionally dependent [12]. Up to our knowledge, a latent class analysis was not used yet for the evaluation of multiple serological tests to diagnose human brucellosis.

The aims of this study were to estimate the true prevalence for brucellosis in two study groups and to evaluate three conditionally dependent serological tests using latent class analysis.

\section{Methods}

\section{Study population, study area, and sampling strategy}

Blood samples of livestock farmers were collected between September 2007 and August 2008 in Mymensingh district. Three hundred and thirty-five livestock owners or hired animal caretakers agreed to participate. The details of the livestock farmers included in this study were described in a previous paper [13]. In brief, out of a total of the 146 unions (sub Upa-Zilla) of Mymensingh district, 28 were randomly selected. One geographical coordinate was randomly selected from each selected union and located by a hand-held GPS reader. Livestock farmers within $0.5 \mathrm{~km}$ radius of the selected point were informed about the survey, and those who agreed were sampled.

Blood samples from prolonged pyrexia (PP) patients were taken randomly once in a week at Mymensingh Medical College (MMC) hospital. These patients originated from Mymensingh and neighboring districts like Netrakona, Jamalpur, Sherpur, and Tangail. Patients with PP were defined having a body temperature higher than $38{ }^{\circ} \mathrm{C}$ for a 3 -week period. Every day, approximately 100 patients visit the outdoor facility of the hospital. Patients who met the inclusion criterion were asked for a blood sample. In addition, hospitalized patients meeting the inclusion criterion at the same day were also asked for a sample. A total of 300 PP blood samples were collected from October 2007 to May 2008.

\section{Collection and handling of blood samples}

The collection and handling of blood samples was described in a previous paper by Rahman et al. [13]. In brief, about $4 \mathrm{~mL}$ of blood was collected with disposable needles and Venoject tubes, labeled, and transported to the laboratory on ice (after clotting) within $12 \mathrm{~h}$ of collection. The blood samples were kept in refrigerator $\left(2-8{ }^{\circ} \mathrm{C}\right)$, and 1 day later, sera were separated by centrifuging at $6000 \mathrm{~g}$ for $10 \mathrm{~min}$.

\section{Serological tests}

All blood samples were tested in parallel by indirect IgG iELISA, RBT, and STAT at the Medicine Department laboratory of Bangladesh Agricultural University, Mymensingh, Bangladesh. RBT was performed as described by Alton et al. [14]. The STAT was carried out on doubling dilutions of serum from 1:20 to 1:320 according to Alton et al. [14]. Brucella abortus and Brucella melitensis antigens (Cypress Diagnostics, Langdorpsesteenweg 160, B-3201, Belgium) were used according to the instruction of the manufacturer. Titres $\geq 1: 160$ were considered as positive. The iELISA was used as described by Limet et al. [15] using B. abortus biotype 1 antigen (Strain Weybridge 99, A epitope). Six dilutions of positive control serum no. 1121 (1/270-1/8340, corresponding to $2-60$ units) were used to generate a standard curve. The detail procedure was described in a previous paper by Rahman et al. [13].

\section{Statistical analysis}

In order to determine the true prevalence, sensitivity, and specificity of the three tests for two subpopulations, Bayesian latent class analysis was performed using a multinomial model, based on conditional probabilities [16]. The full model assuming conditional dependence is overparameterized. It thus requires external (prior) information for prevalence and test characteristics (sensitivity and specificity). Prior information on prevalence [3-5] and sensitivity and specificity of iELISA [17] was extracted from published reports, and three other conditional probabilities adapted by experts of the Department of Infectious and Parasitic Diseases, Faculty of Veterinary Medicine, University of Liège, Belgium (Table 1) were included. The beta prior distribution was considered in Bayesian model. The analysis was conducted in WinBUGS 1.4 [18] and R 3.2.2 (R Foundation and Statistical Computing 2015). The model was run with a burn-in of 50,000 iterations, and estimates were based on a further 50,000 iterations and three chains. The posterior predictive $P$ value, the Deviance Information Criterion (DIC), and the number of parameters effectively estimated by the model $(\mathrm{pD})$ ) were used to assess the fit between the prior information and the test results [16]. The WinBUGS code for conditional dependence of three tests for a two populations Bayesian model is shown in Additional file 1: Appendix A.

The positive predictive value (PPV) and negative predictive value (NPV) of the tests were calculated using Eqs. 1 and 2 in Bayesian model.

$$
\mathrm{PPV}=\frac{\text { Sensitivity }(\mathrm{Se}) * \text { Prevalence }(\mathrm{Pr})}{\operatorname{Se} * \operatorname{Pr}+(1-\text { Specificity }(\mathrm{Sp})) *(1-\mathrm{Pr})}
$$


Table 1 Prior information for the Bayesian latent class evaluation of three serological tests for the diagnosis of brucellosis in livestock farmers and prolonged pyrexia patients (Beta distribution)

\begin{tabular}{ll}
\hline Conditional probabilities & $\begin{array}{l}\text { Prior information } \\
\text { (alpha, beta) }\end{array}$ \\
\hline Prevalence (pr[1] and pr[2] in the model & $(1.57,29.19)$ \\
in Additional file 1: Appendix A) [13-15] & \\
Sensitivity of the iELISA for the diagnosis \\
of sero-positive individuals (th1[2] and \\
th2[2] in the model in Additional file 1: \\
Appendix A[29] \\
Specificity of the iELISA for the diagnosis \\
of sero-negative individuals (th1[3] and \\
th2[3] in the model in Additional file 1: \\
Appendix A) [29] \\
Probability to have a positive result for \\
the RBT if the individual is sero-positive \\
and positive for the iELISA (th1 [4] and \\
th2[4] in the model in Additional file 1: \\
Appendix A) \\
Probability to have a positive result for \\
the STAT if the individual is sero-positive \\
and positive for the iELISA and RBT (th1[8] \\
and th2[8] in the model in Additional file 1: \\
Appendix A) \\
Probability to have a negative result for \\
the STAT if the individual is sero-negative \\
and negative for the iELISA and RBT (th1[12] \\
and th2[12] in the model in Additional file 1: \\
Appendix A)
\end{tabular}

iELISA indirect ELISA, RBT Rose Bengal test, STAT standard tube agglutination test

$$
\mathrm{NPV}=\frac{\mathrm{Sp} *(1-\mathrm{Pr})}{(1-\mathrm{Se}) * \operatorname{Pr}+\mathrm{Sp}(1-\mathrm{Pr})}
$$

\section{Sensitivity analyses}

The influence of the prior information on the estimates of the characteristics of the diagnostic tests was verified using sensitivity analysis. This was done by using uniform priors and slight perturbations (in steps of 10 or $15 \%)$ of the prior intervals [18].

\section{Results}

\section{Descriptive statistics}

Cross-classified results of the three serological tests are shown in Table 2. From the 335 livestock farmers, only $0.6 \%$ were positive and $97.3 \%$ were negative in all three tests. The apparent prevalence of brucellosis among livestock farmers based on a parallel interpretation of the three tests was $2.7 \%$. Of 300 PP patients, only $2.0 \%$ were positive and $97.3 \%$ were negative in all three tests. Based on a parallel interpretation (if positive in at least one test), the apparent prevalence of brucellosis among PP patients was $2.7 \%$; $32.8 \%$ (110/335) of the livestock farmers and $33.7 \%(101 / 300)$ of the PP patients were
Table 2 Cross-classified test results of three serological tests applied on livestock farmers and prolonged pyrexia patients in Bangladesh

\begin{tabular}{lllll}
\hline iELISA & RBT & STAT & Livestock farmers & PP patients \\
\hline 1 & 1 & 1 & 2 & 6 \\
1 & 1 & 0 & 0 & 0 \\
1 & 0 & 1 & 0 & 0 \\
1 & 0 & 0 & 0 & 0 \\
0 & 1 & 1 & 3 & 1 \\
0 & 1 & 0 & 1 & 0 \\
0 & 0 & 1 & 3 & 1 \\
0 & 0 & 0 & 326 & 292 \\
Total & & & 335 & 300
\end{tabular}

1 positive test result, $O$ negative test result, $i E L I S A$ indirect enzyme-linked immunosorbent assay, RBT Rose Bengal Test, STAT standard tube agglutination test, $P P$ prolonged pyrexia

female. All livestock farmers had contact with cattle $(66.0 \%)$ or goats $(17.3 \%)$ or with both $(16.7 \%)$. Among PP patients, only $27 \%(81 / 300)$ had contact with cattle and or goats.

\section{Posterior estimates}

The true prevalence of brucellosis among livestock farmers and PP patients is presented in Table 3. The true prevalence of brucellosis in livestock farmers and PP patients estimated were $1.1 \%$ (95 \% (CrI 0.1-2.8) and $1.7 \%$ (95 \% CrI 0.2-4.1), respectively. The performance of all three tests was similar in both populations. In both groups, specificity of all tests was greater than $97.8 \%$ (95 \% CrI 96.2-99.9). The sensitivity of iELISA, RBT, and STAT varied from $68.1 \%$ (95\% CrI 54.5-80.7) to $69.6 \%$ (95 \% Cr1 56.0-81.6), $79.4 \%$ (95 \% CrI 59.5-95.0) to $79.2 \%$ (95 \% CrI 60.3-94.8), and 80.5 (95\% CrI 63.1-93.8) to 80.6 (95\% CrI 63.6-93.8) in livestock farmers and PP patients, respectively.

Table 3 Estimates of true prevalence, sensitivity, specificity of three serological tests used for the diagnosis of brucellosis in livestock farmers and PP patients in Bangladesh

\begin{tabular}{|c|c|c|c|}
\hline & & Livestock farmer & PP patients \\
\hline Test & Var & Mean $(95 \% \mathrm{Crl})$ & Mean $(95 \% \mathrm{Crl})$ \\
\hline & Prev & $1.1(0.1-2.8)$ & $1.7(0.2-4.1)$ \\
\hline \multirow[t]{2}{*}{ iELISA } & $\mathrm{Se}$ & $68.1(54.2-80.7)$ & $69.6(56.0-81.6)$ \\
\hline & Sp & $98.8(97.7-99.5)$ & $98.4(97.0-99.3)$ \\
\hline \multirow[t]{2}{*}{ Rose Bengal } & $\mathrm{Se}$ & $79.4(59.5-95.0)$ & $79.2(60.3-94.8)$ \\
\hline & Sp & $97.9(96.1-99.3)$ & $98.2(96.4-99.5)$ \\
\hline \multirow[t]{2}{*}{ Standard tube agglutination } & $\mathrm{Se}$ & $80.5(63.1-93.8)$ & $80.6(63.6-93.8)$ \\
\hline & Sp & 97.8 (96.2-98.9) & $97.9(96.4-99.2)$ \\
\hline
\end{tabular}

PP prolonged pyrexia, Var variable, Prev prevalence, $\mathrm{Crl}$ credibility interval, Se sensitivity, $S p$ specificity 


\section{Positive and negative predictive values}

The PPV and NPV of three serological tests were shown in Table 4. The PPV of three serological tests varied from $27.9 \%$ (95 \% CrI 3.6-62.0) to $36.3 \%$ (95 \% CrI 5.6-70.5) in livestock farmers and $39.8 \%$ (95 \% CrI 6.0-75.2) to $42.7 \%$ (95 \% CrI 6.4-83.2) in PP patients. The NPV of all three tests were very high and more than $99.5 \%$.

\section{Results of sensitivity analyses}

The true prevalences and specificities of all three tests obtained from the different models of sensitivity analyses were similar. Whereas the estimated sensitivities varied in two models and yielded wider confidence intervals. But as their $95 \%$ credibility intervals overlap, the observed differences were not statistically important (data not shown).

\section{Discussion}

Data on the true prevalence of brucellosis, characteristics of three serological tests in livestock farmers, and PP patients from Bangladesh are provided.

A Bayesian latent class evaluation was used to estimate the true prevalence of brucellosis in livestock farmers, PP patients, and at the same time to evaluate three conditionally dependent serological tests. Bangladesh has to be considered to be endemic for brucellosis but with a very low prevalence in animals and humans [19]. In areas of low endemicity, the risk for human infection originates either from consumption of non-pasteurized dairy products or occupation threatening veterinarians, abattoir workers, farmers, and laboratory personnel. In this study, it was possible to estimate the true prevalence for livestock farmers. Sample sizes for other occupational groups were too small to do so, and the method of collection was also non-random. This is another limitation of this study. However, livestock farmers are a promising study group as almost $85 \%$ of rural households own animals and $75 \%$ of the population rely to some extent on livestock for their livelihood [20, 21].

Table 4 The positive and negative predictive values of three serological tests

\begin{tabular}{llll}
\hline Test & Variable & $\begin{array}{l}\text { Livestock farmer } \\
(95 \% \text { Crl) } \\
\text { Prevalence: 1.1\% }\end{array}$ & $\begin{array}{l}\text { PP patients } \\
\text { (95\% Crl) } \\
\text { Prevalence: 1.7\% }\end{array}$ \\
\hline iELISA & PPV (\%) & $36.3(5.6-70.5)$ & $41.4(6.6-76.1)$ \\
& NPV (\%) & $99.6(98.9-99.9)$ & $99.5 \%(98.6-99.9)$ \\
Rose Bengal & PPV (\%) & $29.9(3.6-69.5)$ & $42.7(6.4-83.2)$ \\
& NPV (\%) & $99.8(99.2-99.9)$ & $99.6 \%(98.8-99.9)$ \\
Standard tube & PPV (\%) & $27.9(3.6-62.0)$ & $39.8(6.0-75.2)$ \\
agglutination & NPV (\%) & $99.8(99.3-99.9)$ & $99.6 \%(98.9-99.9)$ \\
\hline
\end{tabular}

PPV positive predictive value, NPV negative predictive value, $\mathrm{Crl}$ credibility interval
The true prevalence for this group was estimated to be $1.1 \%$. Brucellosis is a pyrexic disease. As such, it was of interest to investigate also PP patients due to the assumption that brucellosis may be regularly ignored or misdiagnosed. If so, the number of pyrexic patients infected with brucellosis is considered to be valuable information not only for family physicians but also for policy makers. In this study, we focused on PP patients because these patients take antipyretic drugs and antibiotics inappropriate for brucellosis, and see doctors only if recovery does not occur. Among PP patients, $1.7 \%$ were found to be positive for brucellosis which confirms our assumption that brucellosis is ignored or misdiagnosed by physicians in Bangladesh.

Both in livestock farmers and PP patients, the performance of all three serological tests was similar. RBT does not need sophisticated infrastructure or extensive training; it is amazingly cheap and fast. For the Bangladesh setting, RBT is the test of choice. For some endemic countries, authors reported specificity problems of the RBT [22, 23]. In order to overcome this specificity problem, Diaz et al. [24] recommended a modified protocol, i.e., predilution of serum $>1: 4$. Interestingly, we found almost the same performance for the RBT as described by Diaz et al. [24] but without any modification. If the prevalence of a disease is very low as it is in Bangladesh, there will be lower positive and higher negative predictive values for the tests [25]. We have also observed lower positive predictive values of the serologic tests. The highest positive predictive value of RBT in PP patients was $42.7 \%$ indicating that $42.7 \%$ test positive patients truly have the disease and the remaining are falsely positive. The positive predictive value may be increased by applying a second test with high specificity and/or by testing patients having history of exposure with known risk factors like contact with animals, consumption of raw milk, and/or having some symptoms like pyrexia, arthralgia, backache, etc.

Anti-Brucella antibodies, especially IgG, can persist for a longer period of time, i.e., several months even after recovery from disease [26]. For that reason, the presence of anti-Brucella antibodies cannot reflect the true disease status as described above. Thus, diagnosis should be confirmed in a sero-positive patient by the presence of at least one of the clinical symptoms and signs suggestive of brucellosis like pyrexia, arthralgia, headache, backache, hepatomegally, splenomegally, etc. [23, 27]. Applying a more specific test genus or species-specific real time PCR may also be performed [28] to avoid unjustified costs, drug toxicity, and masking of other potentially dangerous diseases like tuberculosis, which are also endemic in Bangladesh.

For a quantitative test, the sensitivity or specificity depends largely on the cut-off value chosen and other factors like endemicity, status and duration of infection, 
persistence of antibody titres after treatment, presence of cross-reacting pathogens etc. [25]. The cut-off value of the iELISA $(\geq 20 \mathrm{U} / \mathrm{ml})$ used in our study seems to be appropriate to avoid false positives as its specificity was very high ranging from 99.3 to $99.6 \%$. WHO and OIE provide guidelines for STAT and RBT standardization, but not for the iELISAs. So, the iELISA test kits provided by different companies are not standardized and it is difficult to compare the results of different studies due to different cutoffs used. In general, a "new" cutoff should be determined under local conditions to avoid false positives.

Like many other authors, we have considered a STAT titre of $1: 160$ as positive $[17,22]$. As already mentioned earlier, in regions where brucellosis is endemic, a large proportion of the population may have persistent Brucella-specific antibody titres. In this scenario, some authors recommend to use STAT titres of 1:320 or higher to avoid false positives [28, 29]. However, in our study, a STAT titre of 1:160 seems to be appropriate as this titre resulted in specificity ranging from 98.2 to $98.8 \%$ indicating good fit for our setting.

The Bayesian latent class evaluation of diagnostic tests requires an assessment of variations in the prior information on the estimated parameters using a sensitivity analysis [30]. Our sensitivity analysis indicated that the use of diffused priors had no relevant influence on the estimated prevalence and test sensitivities and specificities.

\section{Conclusions}

Based on the performance of the three serological tests validated in a setting where the prevalence of brucellosis is low in humans and animals, no single test can be recommended for routine diagnosis of human brucellosis in Bangladesh. Applying a second test with high specificity and/or testing patients with the history of exposure with known risk factors and/or testing patients having some clinical signs and symptoms of brucellosis may increase the positive predictive value of the serologic tests.

\section{Additional file}

Additional file 1: Appendix A. (DOCX $23 \mathrm{~kb})$

\section{Acknowledgements}

The authors are grateful to Professor Dr. Akram Hossain and Dr. Noor Muhammad, Department of Microbiology, Mymensingh Medical College, Bangladesh, for their help in sampling and treatment of cases.

\section{Funding}

This study was supported by the Belgian Directorate General for Development Cooperation. The funding body had no role in the design of the study and collection, analysis, and interpretation of data and in writing the manuscript.
Availability of data and materials

Data and codes for statistical analysis are available in Additional file 1: Appendix A. Raw data is also available on request.

\section{Authors' contributions}

AKMAR and CS conceived of the study and participated in its design. AKMAR carried out serological tests. AKMAR and DB performed statistical analysis. AKMAR, CS, and DB drafted the manuscript. All authors read and approved the final manuscript.

\section{Competing interests}

The authors declare that they have no competing interests.

\section{Consent for publication}

Informed written consent was also taken from all participants to report their demographic information in publications.

\section{Ethics approval and consent to participate}

The study protocol was peer reviewed and cleared for ethics by the Ethical Review Committee of Mymensingh Medical College (MMC). Informed written consent was taken from all individuals prior to blood sample collection.

\section{Author details}

'Department of Medicine, Bangladesh Agricultural University, Mymensingh 2200, Bangladesh. ${ }^{2}$ Research Unit of Epidemiology and Risk Analysis applied to the Veterinary Sciences (UREAR-ULg), Department of Infectious and Parasitic Diseases, Faculty of Veterinary Medicine, University of Liège, Liège, Belgium. ${ }^{3}$ Department of Biomedical Sciences, Institute of Tropical Medicine, Antwerpen, Belgium.

Received: 21 May 2016 Accepted: 10 August 2016

Published online: 04 October 2016

\section{References}

1. Ariza J, Bosilkovski M, Cascio A, Colmenero JD, Corbel MJ, Falagas ME, Memish ZA, Roushan MRH, Rubinstein E, Sipsas NV, Solera J. Perspectives for the treatment of brucellosis in the 21st century: the loannina recommendations. PLoS Med. 2007:4:e317

2. World Health Organization. The control of neglected zoonotic diseases. Geneva: Report of a joint WHO/DFID-AHP, pp 54. 2005. http://www.who.int/ zoonoses/Report_Sept06.pdf. Accessed 28 Sept 2015.

3. Rahman MM, Chowdhury TIMFR, Rahman A, Haque F. Seroprevalence of human and animal brucellosis in Bangladesh. Indian Vet J. 1983;60:165-8.

4. Rahman MM, Haque M, Rahman MA. Seroprevalence of caprine and human brucellosis in some selected areas of Bangladesh. Bangladesh Vet J. 1988:22:85-92.

5. Muhammad N, Hossain MA, Musa AK, Mahmud MC, Paul SK, Rahman MA, Haque N, Islam MT, Parvin US, Khan SI, Nasreen SA. Seroprevalence of human brucellosis among the population at risk in rural area. Mymensingh Med J. 2010:19:1-4.

6. Adel A, Saegerman C, Speybroeck N, Praet N, Victor B, De Deken R, Soukehal A, Berkvens D. Canine leishmaniasis in Algeria: true prevalence and diagnostic test characteristics in groups of dogs of different functional type. Vet Parasitol. 2010;172:204-13.

7. Muñoz PM, Blasco JM, Engel B, de Miguel MJ, Marín CM, Dieste L, Mainar-Jaime RC. Assessment of performance of selected serological tests for diagnosing brucellosis in pigs. Vet Immunol Immunopathol. 2012;146:150-8.

8. Praud A, Gimenez O, Zanella G, Dufour B, Pozzi N, Antras V, Meyer L, Garin-Bastuji B. Estimation of sensitivity and specificity of five serological tests for the diagnosis of porcine brucellosis. Prev Vet Med. 2012;104:94-100.

9. Vacek PM. The effect of conditional dependence on the evaluation of diagnostic tests. Biometrics. 1985;41:959-68.

10. Gardner IA, Stryhn H, Lind P, Collins MT. Conditional dependence between tests affects the diagnosis and surveillance of animal diseases. Prev Vet Med. 2000:45:107-22

11. Nielsen K. Diagnosis of brucellosis by serology. Vet Microbiol. 2002;90:447-59.

12. Dendukuri N, Joseph L. Bayesian approaches to modeling the conditional dependence between multiple diagnostic tests. Biometrics. 2001;7:158-67.

13. Rahman AKMA, Saegerman C, Berkvens D, Fretin D, Gani MO, Ershaduzzaman M, Ahmed MU, Emmanuel A. Bayesian estimation of true prevalence, sensitivity and specificity of indirect ELISA, Rose Bengal test and 
slow agglutination test for the diagnosis of brucellosis in sheep and goats in Bangladesh. Prev Vet Med. 2013;110:242-52.

14. Alton $\mathrm{GG}$, Jones $\mathrm{LM}$, Angus RD, Verger JM. Techniques for the brucellosis laboratory. Paris, France: Institut National de la echerché agronomique (INRA); 1988. p. 112-89.

15. Limet JN, Kerkhofs P, Wijfels R, Dekeyser P. Le diagnostic serologique de la brucellose bovine par ELISA. Ann De Med Vet. 1988;132:565-75.

16. Berkvens D, Speybroeck N, Praet N, Adel A, Lesaffre E. Estimating disease prevalence in a Bayesian framework using probabilistic constraints. Epidemiology. 2006;17:145-53.

17. Gómez MC, Nieto JA, Rosa C, Geijo P, Escribano MA, Muñoz A, López C. Evaluation of seven tests for diagnosis of human brucellosis in an area where the disease is endemic. Clin Vaccine Immunol. 2008;15:1031-3.

18. Haley C, Wagner B, Puvanendiran S, Abrahante J, Murtaugh MP. Diagnostic performance measures of ELISA and quantitative PCR tests for porcine circovirus type 2 exposure using Bayesian latent class analysis. Prev Vet Med. 2011;101:79-88.

19. Islam MA, Khatun MM, Werre SR, Sriranganathan N, Boyle SM. A review of Brucella seroprevalence among humans and animals in Bangladesh with special emphasis on epidemiology, risk factors and control opportunities. Vet Microbiol. 2013;166(3):317-26.

20. Anon. Livestock sector brief of Bangladesh. Food and agriculture organization of the United Nations, 2005. (http://www.fao.org/ag/againfo/resources/en/ publications/sector_briefs/lsb_BGD.pdf). Accessed 20 October 2015.

21. BBS. The Bangladesh Census of Agriculture (rural) 1996, Structure of agricultural holdings and livestock population, vol. 1. Dhaka: Bangladesh bureau of statistics; 2004.

22. Konstantinidis A, Minas A, Pournaras S, Kansouzidou A, Papastergiou P, Maniatis A, Stathakis N, Hadjichristodoulou C. Evaluation and comparison of fluorescence polarization assay with three of the currently used serological tests in diagnosis of human brucellosis. Eur J Clin Microbiol Infect Dis. 2007;26:715-21.

23. Ruiz-Mesa JD, Sánchez-Gonzalez J, Reguera JM, Martin L, Lopez-Palmero S, Colmenero JD. Rose Bengal test: diagnostic yield and use for the rapid diagnosis of human brucellosis in emergency departments in endemic areas. Clin Microbiol Infect. 2005;11:221-5.

24. Díaz R, Casanova A, Ariza J, Moriyon I. The Rose Bengal test in human brucellosis: a neglected test for the diagnosis of a neglected disease. PLoS Negl Trop Dis. 2011;5:e950.

25. Greiner M, Gardner IA. Epidemiological issues in the validation of veterinary diagnostic tests. Prev Vet Med. 2000;45:3-22.

26. Godfroid J, Nielsen K, Saegerman C. Diagnosis of brucellosis in livestock and wildlife. Croat Med J. 2010;51:296-305.

27. Rahman AKMA, Dirk B, Fretin D, Saegerman C, Ahmed MU, Muhammad N Hossain A, Abatih E. Seroprevalence and risk factors for brucellosis in a high-risk group of individuals in Bangladesh. Foodborne Pathog Dis. 2012;9:190-7.

28. Kiel WK, Khan Y. Analysis of 506 consecutive positive serologic tests for brucellosis in Saudi Arabia. J Clin Microbiol. 1987;25:1384-7.

29. Memish ZA, Almuneef M, Mah MW, Qassem LA, Osoba AO. Comparison of the Brucella standard agglutination test with the ELISA IgG and IgM in patients with Brucella bacteremia. Diagn Microbiol Infect Dis. 2002;44:129-32.

30. Navarro E, Segura JC, Castaño MJ, Solera J. Use of real-time quantitative polymerase chain reaction to monitor the evolution of Brucella melitensis DNA load during therapy and post-therapy follow-up in patients with brucellosis. Clin Infect Dis. 2006;42:1266-73.

\section{Submit your next manuscript to BioMed Central and we will help you at every step:}

- We accept pre-submission inquiries

- Our selector tool helps you to find the most relevant journal

- We provide round the clock customer support

- Convenient online submission

- Thorough peer review

- Inclusion in PubMed and all major indexing services

- Maximum visibility for your research

Submit your manuscript at www.biomedcentral.com/submit
Biomed Central 